\title{
Erratum to: Combination therapy with steroids and mizoribine in juvenile SLE: a randomized controlled trial
}

Yuriko Tanaka $\cdot$ Norishige Yoshikawa

Shinzaburo Hattori • Satoshi Sasaki • Takashi Ando •

Masahiro Ikeda • Masataka Honda •

for Japanese Study Group for Renal Disease in Children

Published online: 15 December 2009

(C) IPNA 2009

Erratum to: Pediatric Nephrology

DOI 10.1007/s00467-009-1341-4

Unfortunately, Satoshi Sasaki's affiliation was published incorrectly.

The correct version can be seen below.

Satoshi Sasaki, Department of Pediatrics, Hokkaido University, Hokkaido, Japan

The online version of the original article can be found at http://dx.doi. org/10.1007/s00467-009-1341-4.

\section{Y. Tanaka $(\bowtie)$}

Department of Pediatrics,

Dokkyo Medical University Koshigaya Hospital,

2-1-50 Minamikoshigaya,

Koshigaya City, Saitama, Japan 343-8555

e-mail: yuriko@amy.hi-ho.ne.jp

N. Yoshikawa

Department of Pediatrics,

Wakayama Medical University,

Wakayama, Japan

\section{S. Hattori}

Fundamental Medicine,

Kumamoto Health Science University,

Kumamoto, Japan
S. Sasaki

Department of Pediatrics, Hokkaido University,

Hokkaido, Japan

T. Ando

Department of Economic History,

School of Economics and Management,

Lund University,

Lund, Sweden

M. Ikeda $\cdot$ M. Honda

Department of Pediatric Nephrology,

Tokyo Metropolitan Children's Hospital,

Kiyose City, Tokyo, Japan 